Article

\title{
Smartphone-Based pH Sensor for Home Monitoring of Pulmonary Exacerbations in Cystic Fibrosis
}

\author{
Alexander Sun ${ }^{1}$, Tom Phelps ${ }^{1}$, Chengyang Yao ${ }^{1}$, A. G. Venkatesh ${ }^{1}$, Douglas Conrad ${ }^{2}$ \\ and Drew A. Hall 1,* \\ 1 Department of Electrical and Computer Engineering, University of California, San Diego, La Jolla, CA 92093, \\ USA; acs009@ucsd.edu (A.S.); tphelps4432@gmail.com (T.P.); yaobryce@gmail.com (C.Y.); \\ agvenkatesh@hotmail.com (A.G.V.) \\ 2 Department of Medicine, University of California, San Diego, La Jolla, CA 92093, USA; dconrad@ucsd.edu \\ * Correspondence: drewhall@ucsd.edu; Tel.: +1-858-534-3855
}

Academic Editor: Wan-Young Chung

Received: 10 April 2017; Accepted: 23 May 2017; Published: 30 May 2017

\begin{abstract}
Currently, Cystic Fibrosis (CF) patients lack the ability to track their lung health at home, relying instead on doctor checkups leading to delayed treatment and lung damage. By leveraging the ubiquity of the smartphone to lower costs and increase portability, a smartphone-based peripheral $\mathrm{pH}$ measurement device was designed to attach directly to the headphone port to harvest power and communicate with a smartphone application. This platform was tested using prepared $\mathrm{pH}$ buffers and sputum samples from CF patients. The system matches within $\sim 0.03 \mathrm{pH}$ of a benchtop $\mathrm{pH}$ meter while fully powering itself and communicating with a Samsung Galaxy S3 smartphone paired with either a glass or Iridium Oxide (IrOx) electrode. The IrOx electrodes were found to have 25\% higher sensitivity than the glass probes at the expense of larger drift and matrix sensitivity that can be addressed with proper calibration. The smartphone-based platform has been demonstrated as a portable replacement for laboratory $\mathrm{pH}$ meters, and supports both highly robust glass probes and the sensitive and miniature $\mathrm{IrOx}$ electrodes with calibration. This tool can enable more frequent $\mathrm{pH}$ sputum tracking for $\mathrm{CF}$ patients to help detect the onset of pulmonary exacerbation to provide timely and appropriate treatment before serious damage occurs.
\end{abstract}

Keywords: biosensor; Cystic Fibrosis; pH; point-of-care; smartphone; Iridium Oxide

\section{Introduction}

Cystic Fibrosis $(\mathrm{CF})$ is an inherited chronic disease that adversely affects the lungs and digestive systems of about 30,000 children and adults in the United States, as well as approximately 70,000 people worldwide [1]. Due to the abnormally viscous secretions in their lungs and pancreas that lead to inflammation and organ damage, $\mathrm{CF}$ patients often suffer from chronic lung disease, lung infection, bronchiectasis, and malnutrition [2]. While there is currently no cure for $\mathrm{CF}$, fortunately, advances in both screening and treatment have drastically increased the average lifespan and improved their quality of life. However, those with CF are still constantly at risk of pulmonary exacerbations, which can cause irreversible, life shortening destruction of the airways, with obstructive lung disease being the primary cause of morbidity and mortality $[1,3]$. Hence, people living with this lifelong disease must follow a regular treatment routine to stay healthy and maintain optimal lung function, requiring frequent physician visits to test and clear airway infections.

Unfortunately, current tools, such as clinical culturing, take days to obtain results, and are not able to detect whether a patient is undergoing an exacerbation, leaving physicians to make choices about antibiotic treatment based on trial and error. Recent studies have shown that a decrease in $\mathrm{pH}$ of exhaled breath condensate, as well as increase in fermentation products and upregulation of 
protein biomarkers in sputum, is associated with and precede exacerbation events [4-9]. Therefore, monitoring $\mathrm{pH}$ levels as well as other biomarkers over time would allow patients and physicians to make informed decisions about how to treat airway infections before exacerbations progress and cause further damage.

While very few CF patients have the flexibility and means to participate in frequent advanced diagnostic tests for airway infections typically only available in ERs or doctors' offices, almost all have access to smartphones. Hence, in this paper, we present one of the first low-cost, point-of-care (POC) smartphone-based medical tools for at-home monitoring of CF exacerbations that provides physicians with long-term actionable data to adjust therapy. The entire platform (Figure 1) consists of a smartphone, an electronic module that conducts electrochemical $\mathrm{pH}$ measurements, and a sample holder mounted on top of a modified screen-printed electrode. The module plugs into the audio jack of a smartphone, making it hardware compatible with all makes and models and able to extract power directly from the phone obviating the need for a battery or external power source. This device allows CF patients to track their condition daily from home, thus greatly increasing the specificity with which exacerbations can be tracked and treated. Furthermore, their critical information can be collected and sent to medical professionals who can then adjust the treatment regime for infections. The smartphone also incorporates validated quality of life questionnaires, such as the UCSD Shortness of Breath Questionnaire (SOBQ) and the CF Questionnaire Revised (CFQR) to correlate disease activity with the quantitative data collected [10-13]. This peripheral is also intended to be a platform device that, with slight modification, can easily incorporate further electrochemical sensors for other $\mathrm{CF}$ biomarkers, the assays for which are in the progress of being developed.

(a)

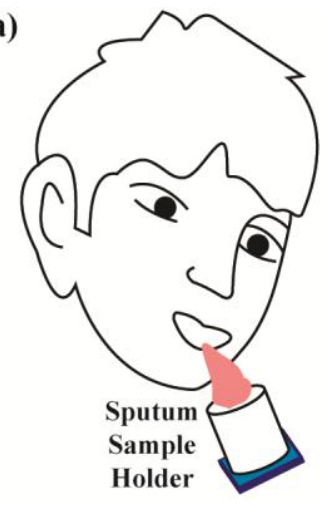

(b)

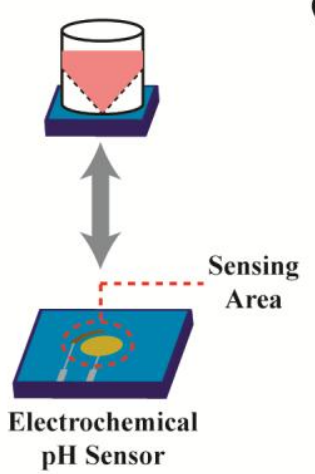

(c)

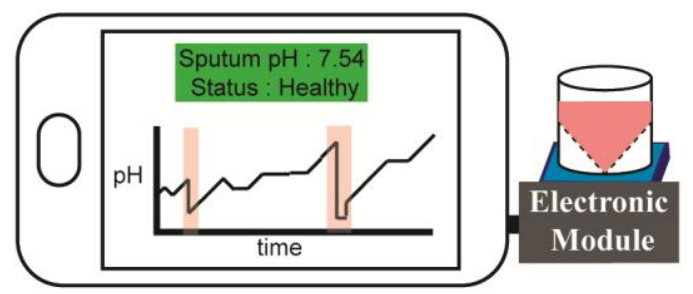

Figure 1. Overview of the smartphone-based $\mathrm{pH}$ sensor platform with (a) sputum collection via a sample holder, the bottom of which is $(\mathbf{b})$ a reusable electrochemical $\mathrm{pH}$ sensor. (c) The entire holder attaches to an external electronic module that plugs directly into the headphone port of the smartphone to make and track measurements via a custom app.

\section{Related Work}

While there have been many sensitive and state-of-the-art POC-oriented biosensors for Cystic Fibrosis, these devices tend to focus solely on the diagnosis of the disease, rather than monitoring the health and detecting the presence of infection in those already diagnosed [14-18]. Since the platform presented here is unique in that it is meant to be a much more frequently used tool to track a $\mathrm{CF}$ patient's daily $\mathrm{pH}$ levels, rather than a one-time use test, the portability of the device is crucial to its operation and overall practicality. While other POC devices meant for diagnosis via immunoassays use microliter volumes of invasive samples such as drops of blood or serum, which often require advanced microfluidics to handle and process $[18,19]$, the specific application targeted in this work allows for the use of sputum, which can be non-invasively extracted in larger volumes $(>1 \mathrm{~mL})$. Hence, a much simpler collection scheme can be employed to handle the biological sample. Furthermore, an electrochemical method of detection is used since the hardware required is comparatively more 
scalable than other methods. For example, there are several smartphone-based $\mathrm{pH}$ meters that use optical measurement techniques exploiting the internal camera and flash of the smartphone [20-25]. These systems are typically prone to interference from environmental lighting or depend on the precision of colorimetric test strips, which are often only intended to give a rough estimate of $\mathrm{pH}$. Achieving high sensitivity requires external lenses, filters, and light sources-all of which are difficult to miniaturize in terms of size and power when compared to electrochemical sensing methods. However, one of the major challenges in electrochemical $\mathrm{pH}$ measurements is the selection of the electrode type, where small size, fabrication difficulty, sensitivity, and stability are traded off with each other [26,27].

While large glass fritted electrodes are still common in laboratory use, there are currently multiple options for state-of-the-art electrochemical $\mathrm{pH}$ sensors more suitable for point-of-care applications such as metal oxide film, conducting polymer, and ion selective field effect transistor (ISFET) electrodes [28-31]. Metal oxides including tungsten, platinum, lead, iridium, ruthenium, and antimony oxides are extremely robust and can withstand harsh environments, high temperature, and high pressure. Specifically, Iridium Oxide (IrOx) has been demonstrated to have a higher sensitivity and faster response time than that of glass [32-34]. Conducting polymers such as polyaniline exhibit less instability and drift overtime than metal oxide electrodes and, hence, require less frequent calibration for comparable accuracy [30,35]. ISFETs can dramatically reduce the size and area of sensors, but, due to their fabrication cost and complexity, they are more appropriate for applications requiring nanoscale or arrayed sensors rather than for instances where macroelectrodes would suffice [28,31].

On the electronics side, ever since the audio-jack was first demonstrated as a viable communication and power transfer port on the smartphone [36], many health-related sensors have been similarly integrated with the smartphone to enable novel mobile health tools [27,37-39]. However, the intrinsically low and inconsistent maximum output power of different headphone ports limits the types of sensors that can leverage the harvesting technique to those with low enough power consumption. Furthermore, most harvesting designs, including commercial ICs (e.g., NXP OM13069), settle for suboptimal power transfer efficiency across different makes and models of smartphones, further lowering the power available to the sensing electronics and reducing the device's overall compatibility with different host devices [40]. Hence, many of these sensors that require extensive power often use an external battery minimizing the advantage of using the headphone port in the first place. This work, instead, is based on previous augmented power harvesting designs that address this issue through implementing automatic power transfer efficiency optimization circuits and algorithms. In the following sections, our design of a more efficient power harvesting electrochemical platform coupled with an inexpensive and reliable Iridium Oxide (IrOx) electrode is discussed and addresses many of these issues.

\section{Design of a Smartphone-Based Sensor Platform}

The smartphone-based electrochemical sensing platform discussed below is based on a potentiostat developed previously with modifications made to tailor the device for POC $\mathrm{pH}$ sensing [41]. The platform consists of three main items: (1) the peripheral electronic module, (2) a point-of-care oriented $\mathrm{pH}$ sensor, and (3) the smartphone application, which communicates with and manages the data collected from the sensor.

\subsection{Smartphone Peripheral $p H$ Sensor Module}

The peripheral module (Figure 2) plugs directly into the smartphone's headphone jack to harvest power and facilitate bidirectional communication between the phone and the module. By using the headphone port instead of other interfaces available on the smartphone (e.g., USB or Lightning port), the module can work across all types of smartphones, even tablets and some feature phones, regardless of the make or model. Furthermore, since power can be efficiently harvested from the headphone jack, the user does not have to deal with the hassle of charging, managing, or replacing additional batteries common in devices that use Bluetooth. Even though the AC coupled nature of the audio port requires 
additional circuitry in order to utilize the phone's energy, this harvesting method has been shown to provide enough power for similar low-power sensors with small form factors.
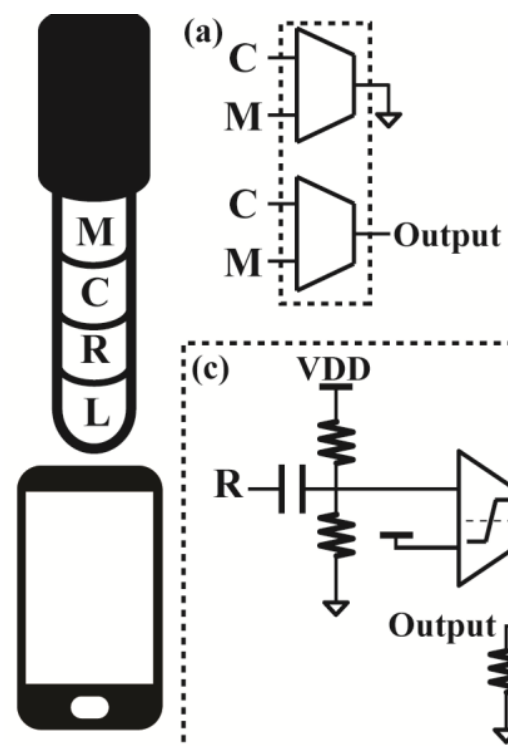

(b)

\section{Impedance Matched Power Harvester}

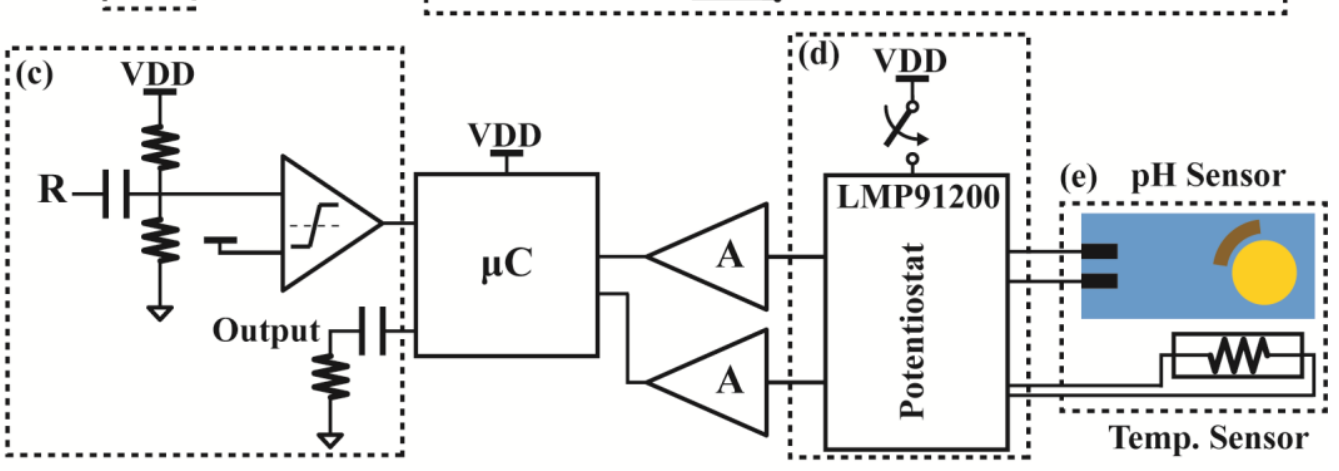

Figure 2. Block diagram of the electronic $\mathrm{pH}$ module that communicates with the smartphone and interfaces with the sensors. The module contains: (a) multiplexers to select the correct pins to connect to the common and microphone ports, (b) an impedance matched power harvester that rectifies and transforms a signal from the left audio output to power the entire module, (c) bidirectional AC coupled communication with the smartphone that sends back the gathered data, a (d) readout and signal conditioning circuitry, and (e) $\mathrm{pH}$ and temperature sensors.

This module also contains an analog front-end that handles measuring data from the sensors. While the actual mechanism of electrochemical $\mathrm{pH}$ sensing varies depending on the type of electrode used, such as standard glass electrodes, ion selective field-effect transistors (ISFET), or oxide-film electrodes, the measurement method is still the same in all cases. Due to the presence of hydrogen ions inside a test solution, a potential difference builds up between two electrodes submerged in the solution that have been modified to specifically detect hydrogen. By measuring this voltage without disturbing the electrochemical cell, the $\mathrm{pH}$ level of the solution can be determined. Finally, an on-board microcontroller relays the information back to the smartphone for analysis. Due to the AC coupling, a frequency modulation technique is used to transmit and receive data.

\subsubsection{Efficient Power Harvesting}

The smartphone's audio port consists of four connections: left $(\mathrm{L})$ and right $(\mathrm{R})$ audio outputs, microphone $(\mathrm{M})$ input, and common $(\mathrm{C})$. The AC coupling restricts the module from directly tapping into the DC power of the audio output. Hence, to provide power, the smartphone outputs a sinusoidal tone at a single adjustable frequency $(5-20 \mathrm{kHz})$ on the left channel. The module boosts this low voltage $(\sim 200 \mathrm{mV})$ tone with a transformer, passes it through a voltage rectifier, and then steps it up to $3.3 \mathrm{~V}$ with a DC-DC converter to power the rest of the board.

Different smartphones, however, have different audio driver characteristics meaning that the output impedance and power available from the headphone port can vary. This inconsistency can cause poor impedance matching between the different phones and the module leading to inefficient power transfer and inadequate power delivered to the module. To account for this variation, an impedance matching network is introduced into the power harvesting circuitry that can be tuned by adjusting the frequency of the tone provided by the smartphone. Hence, regardless of the smartphone used, the module can adjust its own impedance to match the output impedance of the smartphone, 
thereby always providing optimum power transfer to the module, improving the efficiency from $\sim 50 \%$ up to $85 \%$ of the available power [40]. This architecture has also been shown to harvest $3 \times$ more power than a commercial off-the-shelf chip using the same phone [42].

\subsection{2. $\mathrm{pH}$ and Temperature Measurements}

A low-power, small form-factor, commercial, analog front-end (LMP91200, Texas Instruments, TX, USA), which can interface with a variety of $\mathrm{pH}$ electrodes, was chosen to measure the potential difference that develops between the two terminals of the electrode in the test solution. Essentially, the component contains a high input impedance buffer $(\sim 100 \mathrm{~T} \Omega)$ that can probe the voltage of the electrochemical cell, which has a large impedance $(\sim 100 \mathrm{M} \Omega)$. The output of the buffer is amplified via a non-inverting amplifier (OPA2333, Texas Instruments, TX, USA) with a gain of 5 and read by the 8-bit analog to digital converter (ADC) inside the microcontroller (PIC16F690, Microchip, Chandler, AZ, USA).

It is desirable for the module to be able to use different types of $\mathrm{pH}$ electrodes, each with varying voltage ranges, to compare multiple electrodes. To accommodate this large variation of voltage ranges while still maximizing the accuracy of the measurement circuitry, the common-mode voltage of both the $\mathrm{pH}$ electrode and the amplifier can be adjusted for each different type of electrode. Furthermore, since the $\mathrm{pH}$ measurements are highly dependent on the temperature, a thermistor biased by a current source on the LMP91200 is dipped into the solution under test to measure its temperature, which is used to compensate the $\mathrm{pH}$ data for any thermal shifts that occur. By switching from the onboard thermistor to an external sensor via a $2.5 \mathrm{~mm}$ audio port, the module is also compatible with $\mathrm{pH}$ electrodes integrated with internal temperature sensors. The smartphone can then easily use the temperature readings and the Nernst equation to calibrate the $\mathrm{pH}$.

\subsubsection{Communication via Frequency Modulation}

Another discrepancy that exists between some smartphone models is that the microphone and common terminals are sometimes swapped in certain phones, such as the Motorola Moto X. To account for this variation, multiplexers are placed on both the microphone and common channel to be able to interchange them if necessary. Since the microphone pin will be at a higher voltage than the common pin due to the presence of the current source at the microphone output, a comparator tied to both allows the module to determine the correct orientation automatically. Once the microphone channel has been properly set, communication can take place between the device and its host.

However, the AC coupling of both the microphone and audio channels blocks low frequency signals from being transmitted between the smartphone and the module. While simply sending packets of data via bit banging or a UART has been demonstrated to work in short bursts, these methods typically suffer from reliability issues. Distortion of these data packets originates from the signal dependent shaping of the waveform that occurs on the audio channels causing drift and amplitude variation. To avoid this effect, packets are instead constantly modulated using frequency shift keying (FSK) as shown in Figure 3, where the frequency component of the signal holds the data. Hence, this modulation scheme remains robust even in the presence of shifts in the DC voltage or attenuation of the signal amplitude. To minimize the power requirement of the microcontroller, low $1.2 \mathrm{kHz}$ and $2.2 \mathrm{kHz}$ frequency signals are used to represent " 0 " and " 1 ", respectively. This implementation achieves a bit rate of 300 bits per second, which is higher than is necessary due to the low data rate nature of the sensors. 


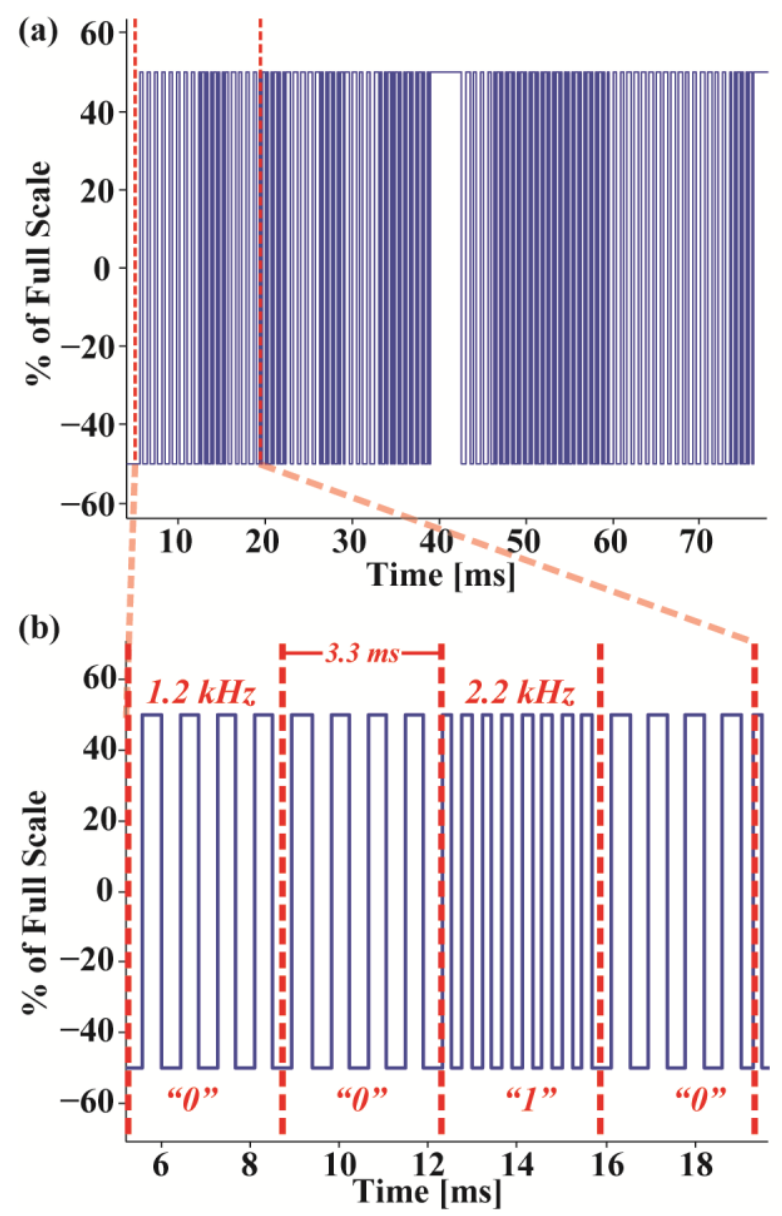

Figure 3. (a) A packet with frequency shift keying modulation and (b) a portion of the data decoded back to its original bits with zeros at $1.2 \mathrm{kHz}$ and ones at $2.2 \mathrm{kHz}$.

\subsection{Point-of-Care Oriented $p H$ Sensor}

Since several types of the potentiometric $\mathrm{pH}$ sensors discussed earlier would each be useful under different scenarios and conditions, instead of designing the platform around a specific type of sensor, the device in this work is meant to be compatible with a wide range of potentiometric $\mathrm{pH}$ sensors. This strategy also allows the device to incorporate ongoing improvements in ion-selective sensors through replacement of the electrode without a full platform redesign. In this work, both standard glass electrodes and Iridium Oxide modified screen-printed electrodes are used to demonstrate the platform's effectiveness as a POC sensor and its compatibility with multiple types of $\mathrm{pH}$ sensors. The glass $\mathrm{pH}$ probe was chosen since, due to its excellent sensitivity, selectivity, stability, and long lifetime, it is the gold standard used in laboratory settings to determine the $\mathrm{pH}$ of an aqueous solution [43]. However, the semi-permeable glass membrane that gives the probe these properties is very fragile, expensive to fabricate, and difficult to miniaturize for POC applications. Iridium oxide was selected for the POC oriented sensor because it is a well-established and thoroughly studied sensor type allowing us to obtain a highly sensitive and stable electrode with simple and inexpensive fabrication steps. IrOx electrodes built on sputtered metal or wire (gold or platinum) have been shown to have high sensitivity $(63.5 \mathrm{mV} / \mathrm{pH}), 0.02 \mathrm{pH}$ accuracy, relatively long lifetime ( 1 month to years), and low drift (0.1 mV/day) [32,33,43]. Furthermore, IrOx film is often used to coat invasive neural probes implanted under the skin or directly in the brain, with extensive research demonstrating that IrOx is highly biocompatible [44-47]. Hence, $\mathrm{IrOx}$ is a safe and ideal electrode material for at-home $\mathrm{pH}$ monitoring, especially since the electrode surface in this application does not come into direct contact 
with the user. Additionally, to make these electrodes easier and less expensive to fabricate, commercial gold screen-printed electrodes (SPE) were used as the base metal before modifying with IrOx. SPEs are self-contained as they have both the working and reference electrode on the same substrate and are already mass produced.

\section{Materials and Methods}

A $3.8 \mathrm{~cm} \times 5.8 \mathrm{~cm}$ printed circuit board $(\mathrm{PCB})$ of the electronic module described previously was constructed. Assembly consists of soldering the components onto a custom printed circuit board, programming the on-board microcontroller, and mounting the electronics in a case. The device has standard BNC, 2.5- $\mathrm{mm}$, and 3.5- $\mathrm{mm}$ connectors to attach both glass $\mathrm{pH}$ electrodes and the screen-printed electrodes as well as their corresponding temperature sensors (Figure 4). For all calibration and comparison testing, both standard pH solutions (Orion 910104, 910107, 910110, Thermo Fischer Scientific, Waltham, MA, USA) and phosphate buffered solutions (PBS) at different pH levels were used. A benchtop pH meter (Orion Star A211, Thermo Fischer Scientific, Waltham, MA, USA) measured the actual $\mathrm{pH}$ of each solution and set the nominal level for each.

(a)

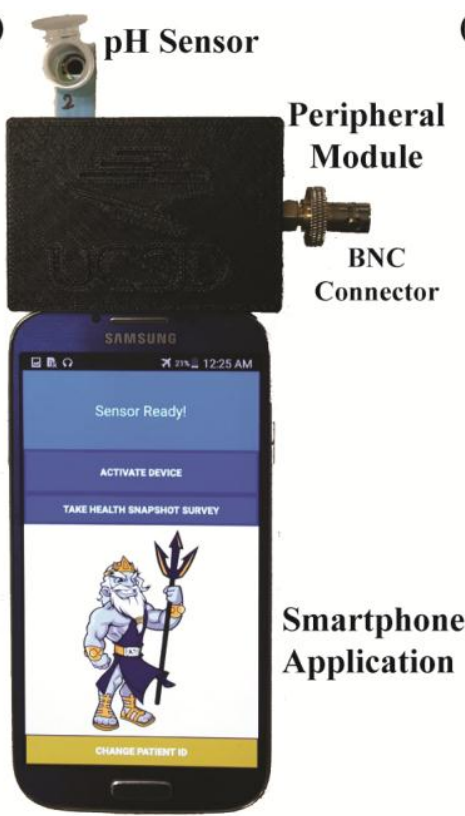

(b) Glass pH Electrode

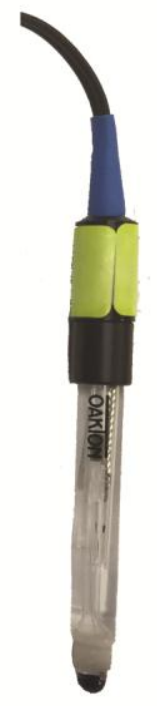

(c)

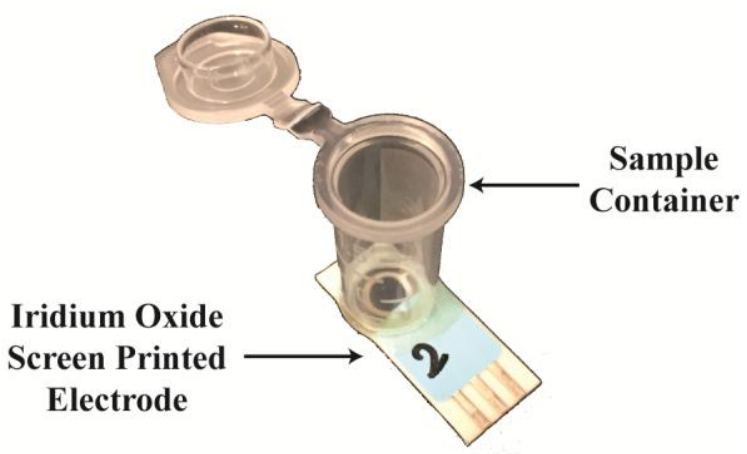

Figure 4. (a) Entire platform with the peripheral module inside a 3D-printed case, $\mathrm{pH}$ sensor chip, and smartphone running the custom $\mathrm{CF}$ application. Both (b) standard glass $\mathrm{pH}$ electrodes and (c) Iridium Oxide modified screen-printed electrodes can be used with this system.

\subsection{Preparation of Iridium Oxide Electrodes}

The Iridium Oxide film was electrodeposited onto gold SPEs (250AT, DropSens, Asturias, Spain) using an established deposition solution [33]. The solution consists of $4.5 \mathrm{mM}$ Iridium tetrachloride (\#516996, Sigma-Aldrich, St. Louis, MO, USA) with 0.3\% hydrogen peroxide (\#H325, Thermo Fischer Scientific, Waltham, MA, USA) and $40 \mathrm{mM}$ oxalic acid dehydrate (\#75699, Sigma-Aldrich, St. Louis, MO, USA). This working solution was left to stabilize at room temperature for two days. To electrodeposit the film, a $50 \mu \mathrm{L}$ droplet was applied to each SPE and scanned with cyclic voltammetry from -0.1 to $1 \mathrm{~V}$ at $20 \mathrm{mV} / \mathrm{s}$ for 25 cycles, roughly $45 \mathrm{~min}$. A commercial potentiostat $(750 \mathrm{E}, \mathrm{CH}$ Instruments, Austin, TX, USA) was used to conduct the cyclic voltammetry deposition. After rinsing in deionized (DI) water, the electrodes were conditioned in a neutral $\mathrm{pH}$ tris buffer for 2 days before use. Each of these electrodes were calibrated using the standard $\mathrm{pH}$ buffers. Between different tests, the electrodes 
were rinsed with DI water, air dried, and kept in a petri dish at room temperature to simulate practical storage conditions.

\subsection{Validation of Module and Iridium Oxide Electrode}

After a single calibration using standard $\mathrm{pH}$ buffers, the electronic module powered and controlled by a Samsung Galaxy S3 smartphone paired with the Oakton pH Probe (WD-35811-74, Oakton Instruments, Vernon Hills, IL, USA) was used to measure the PBS solutions at different pH levels from $\mathrm{pH} 5.9$ to 8.08. Repeated measurements were made daily over the course of a week without recalibration and compared against the nominal $\mathrm{pH}$ set by the commercial $\mathrm{pH}$ meter and probe. Similarly, the IrOx electrodes were used to measure two sets of buffers across 5 days. The solutions were tested with the same setup once a day for an entire week without recalibration with each electrode stored dry.

\subsection{Patient Sputum Samples}

Sputum samples were collected from three CF patients having undergone sputum induction using a standard nebulizer with hypertonic saline. Soon after the samples were gathered, each was initially measured using the smartphone-based $\mathrm{pH}$ platform coupled with a glass electrode to track the change in $\mathrm{pH}$ and temperature over time. Each sample was then measured with both the glass electrode and IrOx electrodes using the smartphone platform as well as the $\mathrm{pH}$ meter. None of these samples were diluted or processed before testing. For calibration of the IrOx electrodes, $200 \mu \mathrm{L}$ aliquots of a single sputum sample were repeatedly spiked with $1 \mu \mathrm{L}$ volumes of $\mathrm{NaOH}$ and $\mathrm{HCl}$ concentrations between $100 \mu \mathrm{M}-1 \mathrm{mM}$ diluted in DI water. Through this addition of the strong acid and base and by using the glass probe to set the nominal values, the $\mathrm{pH}$ of the sputum medium was artificially adjusted to obtain a calibration curve for the IrOx electrodes. In total, no more than $10 \mu \mathrm{L}$ of either acid or base was added to each aliquot, thus limiting error from dilution of the sputum.

\section{Results and Discussion}

\subsection{Performance of Electronic Module}

The electronic module consumes a total maximum power of $6.2 \mathrm{~mW}$ during its measurement operation ( 120 s) with the microcontroller and analog-front-end dominating this number. Assuming that a typical smartphone battery has a capacity of $\sim 1500 \mathrm{mAh}$, even the electronic module running a few times a day would have a negligible effect on the phone's battery life. The total integrated noise $(0.1-10 \mathrm{~Hz})$ of the analog-front-end including the amplifier is $70 \mu \mathrm{V}_{\mathrm{RMS}}$, which sits below the quantization levels of the ADC $(\sim 3.6 \mathrm{mV})$.

Measured $\mathrm{pH}$ data taken by the smartphone-based sensor of all the PBS solutions across several days are averaged and plotted in Figure 5a. The average difference between the measured and the nominal $\mathrm{pH}$ values across all solutions is 0.022 . The standard deviation of measurements of a single solution across all seven measurements is approximately $0.026 \mathrm{pH}$. Hence, when using the glass electrode, the smartphone-based $\mathrm{pH}$ platform matches within $\sim 0.03 \mathrm{pH}$ of the benchtop $\mathrm{pH}$ meter over the timespan of a week without daily recalibration. Not only does this show that the smartphone-based tool matches well with the benchtop meter, but also it suggests that, when using glass probes, frequent or full calibration is not required. 
(a)

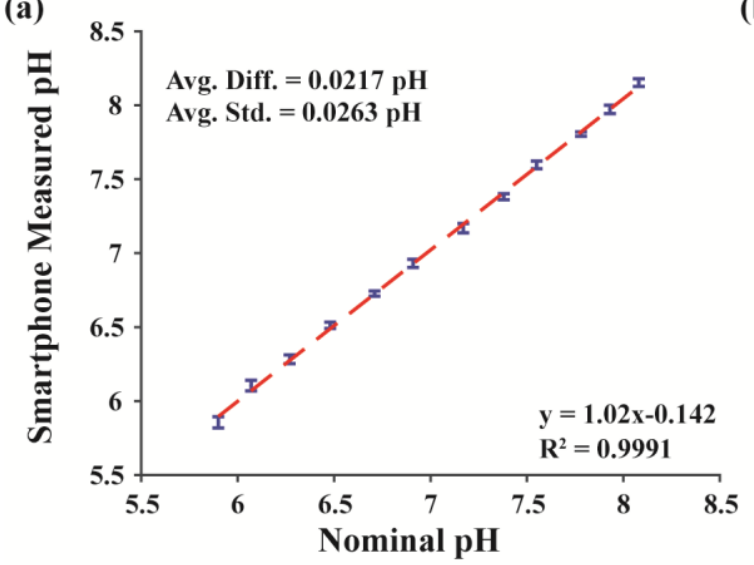

(b)

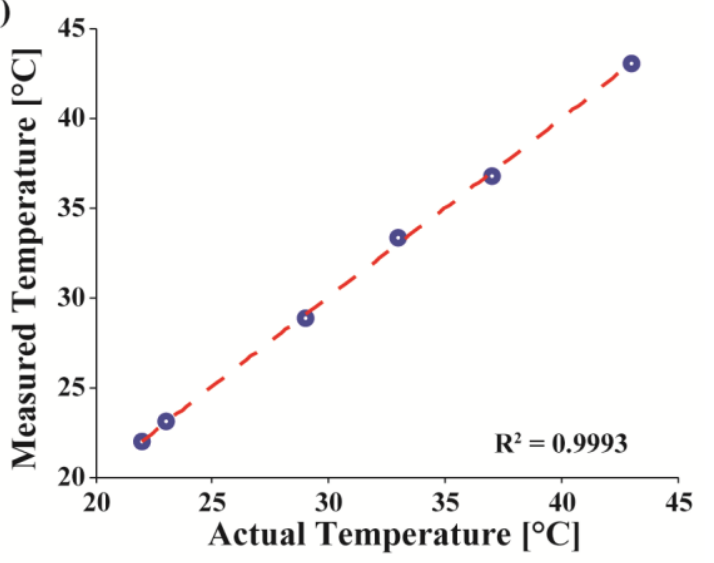

Figure 5. (a) Averaged measured $\mathrm{pH}$ data taken over one week $(n=7)$ for different nominal $\mathrm{pH}$ solutions ranging from 5.9 to $8.08 \mathrm{pH}$. The dotted line is a linear fit of the averaged data; (b) Temperature measured by the module compared to actual temperature recorded by a commercial thermometer from 20 to $45^{\circ} \mathrm{C}$.

To test the onboard temperature sensing, the module's thermistor, after a three-point calibration, was placed on top of a hot plate and measured at various other temperatures from 20 to $45{ }^{\circ} \mathrm{C}$ using the smartphone platform. This data (Figure $5 \mathrm{~b}$ ) was compared to measurements made by a standard commercial IR thermometer (Lasergrip 1080, Etekcity, Anaheim, CA, USA) with an average temperature difference of $0.15^{\circ} \mathrm{C}$. While temperature was used to calibrate the $\mathrm{pH}$ measured by both the $\mathrm{pH}$ meter and the module (Figure S2), temperature calibration was often found to be unnecessary since the environmental conditions and measurement procedures used were kept constant for many of these experiments.

\subsection{Iridium Oxide Electrodes}

The calibration curve from 4-10 $\mathrm{pH}$ of the IrOx electrode plotted with other electrodes in Figure 6a demonstrates the voltage ranges and sensitivity in $\mathrm{mV}$ per $\mathrm{pH}$ of each electrode. As expected, the bare gold electrode has the worst sensitivity, while the glass and IrOx perform well. The IrOx electrodes have a higher sensitivity than that of glass with a $25 \%$ steeper slope.

(a)

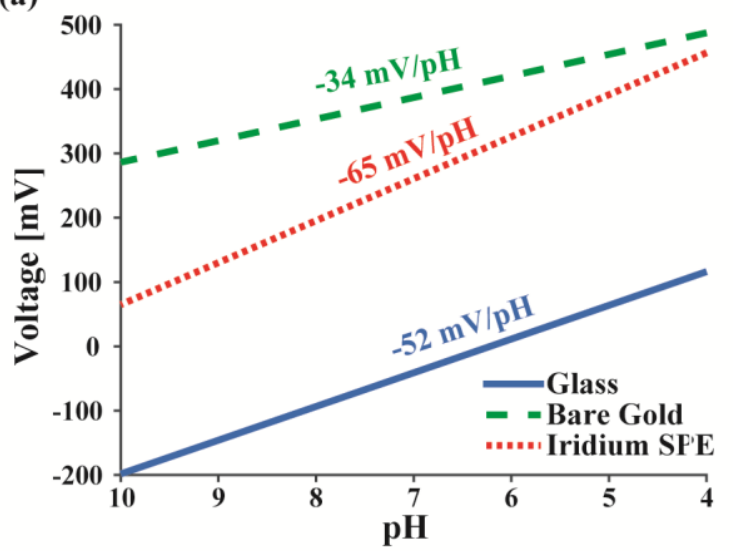

(b)

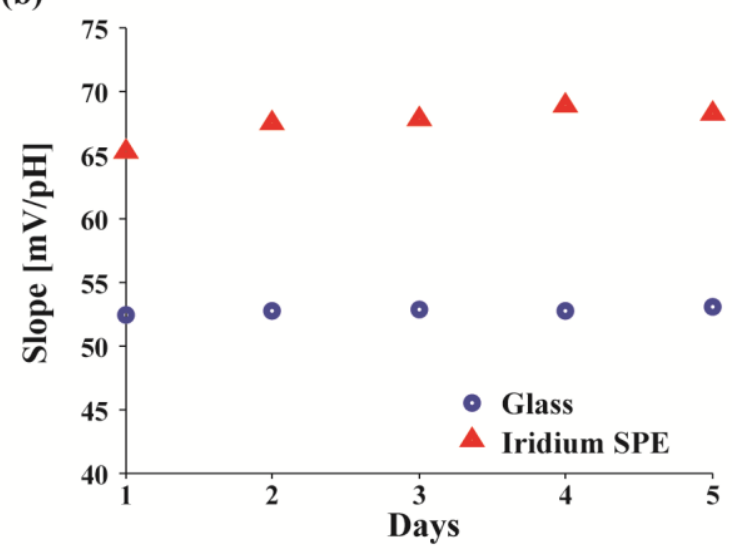

Figure 6. (a) Calibration curve of glass, bare gold SPE, and Iridium-Oxide electrode; (b) pH slope drift over several days for different electrode types. 
One concern with IrOx film electrodes is that their absolute voltage response changes between samples containing varying amounts of salts (i.e., the sample matrix). Testing with both a set of commercial $\mathrm{pH}$ solutions and a set of $\mathrm{pH}$-adjusted $\mathrm{PBS}$ solutions that contain higher concentrations of $\mathrm{KCl}$ and $\mathrm{NaCl}$, the glass electrodes and IrOx SPEs vary on average 0.04 and $0.52 \mathrm{pH}$ in the $6-8 \mathrm{pH}$ range, respectively between the two sets. Hence, when moving to a different buffer, these IrOx SPEs need to be recalibrated. Once properly calibrated with standard solutions, another possible issue that can arise is the drift of electrode's response over time after storage and repeated use. Figure $6 \mathrm{~b}$ shows the variation of the slope $(\mathrm{mV} / \mathrm{pH})$ of the IrOx SPE compared to that of glass. The slopes of IrOx SPEs vary a maximum of $5.57 \%(3.64 \mathrm{mV} / \mathrm{pH})$ over the 5-day period compared to the $1.1 \%(0.56 \mathrm{mV} / \mathrm{pH})$ of the glass probe. Hence, by using a single point pre-test calibration with a standard buffer, both electrodes are relatively stable with drifts of approximately $0.004 \mathrm{pH} /$ day and $0.032 \mathrm{pH} /$ day for the glass probe and IrOx SPE, respectively. Further reliability improvements can be made by introducing a multi-point calibration every few days.

\subsection{Live Sputum Test}

The settling time plot (Figure 7a) of both the $\mathrm{pH}$ and temperature of a sputum sample measured immediately after collection with a glass probe and temperature sensor demonstrates that it takes at least $10 \mathrm{~min}$ for the sample to reach equilibrium. In the standard buffer solution tests discussed previously and seen in the $\mathrm{pH}$ over time plot (Figure $7 \mathrm{~b}$ ), which was recorded immediately after the buffer sample was applied, the IrOx electrodes take roughly $5 \times$ longer to settle than the glass electrode, extending measurement time from $20 \mathrm{~s}$ to $2 \mathrm{~min}$. While the increased measurement time is not ideal for point-of-care applications, due to the temperature change of collected sputum, the settling speed is still fast enough to not affect the overall length of actual sputum testing. Being much longer than the response time of the IrOx electrodes, this settling time with the sputum sets the lower bound on how long the samples need to be incubated on the sensors surface for reliable measurements. Hence, the longer settling time of the IrOx electrodes does not affect the duration of the actual sputum assay. The intrinsic settling time of the IrOx electrodes deviates from that of others found in literature, where these electrodes often reach the final $\mathrm{pH}$ as quickly as or faster than standard glass electrodes. One likely explanation is that the screen-printing method used to fabricate these commercial gold electrodes results in both slower electron transfer kinetics and a vastly different oxide film formation on the electrode surface, which greatly affects its performance and characteristics.

Since sputum will contain different constituents than those of the standard buffers used for characterizing the system, to properly calibrate the IrOx electrodes, a single sputum sample was spiked to different $\mathrm{pH}$ levels to create a calibration curve (Figure 7c). The spiked sputum data not only allow the IrOx sputum data to be mapped back to the actual $\mathrm{pH}$ values, much like the initial calibration curves in standard $\mathrm{pH}$ buffer, but also demonstrate that the response of the $\mathrm{IrOx}$ electrodes to $\mathrm{pH}$ is still approximately linear. Hence, while the response of the IrOx electrodes in sputum do not coincide exactly with that of the laboratory buffers, the relative voltage change is approximately the same in both mediums, so, even without full calibration, the $\mathrm{pH}$ change of a patient's sputum over time still offers accurate and compelling data.

The $\mathrm{pH}$ measurements of each sample with all four combinations of electrodes and tools are shown in Figure $7 \mathrm{~d}$. As expected, there is a large discrepancy between the glass probe and the IrOx electrodes both calibrated with just the standard buffers. After using the spiked sputum data discussed above to adjust results, both sets of data match well with an average difference between the glass probe and IrOx electrodes of $0.067 \mathrm{pH}$. Further improvements can be made by fitting the calibration curve to a higher order polynomial than the simple linear fit used. These results also suggest that the sputum matrix is electrochemically similar between different patients since the calibration curve from a single sputum sample can recover the correct $\mathrm{pH}$ for all three samples without recalibrating for each patient. While the glass probe is still the go to $\mathrm{pH}$ electrode when absolute $\mathrm{pH}$ accuracy and consistency between different buffer types are desired, the IrOx electrodes are valuable in cases where 
the medium of the test solution does not change and miniature, inexpensive, and disposable sensors are favored over the bulky, fragile, and costly glass probes, such as for at-home sputum $\mathrm{pH}$ monitoring for CF patients.
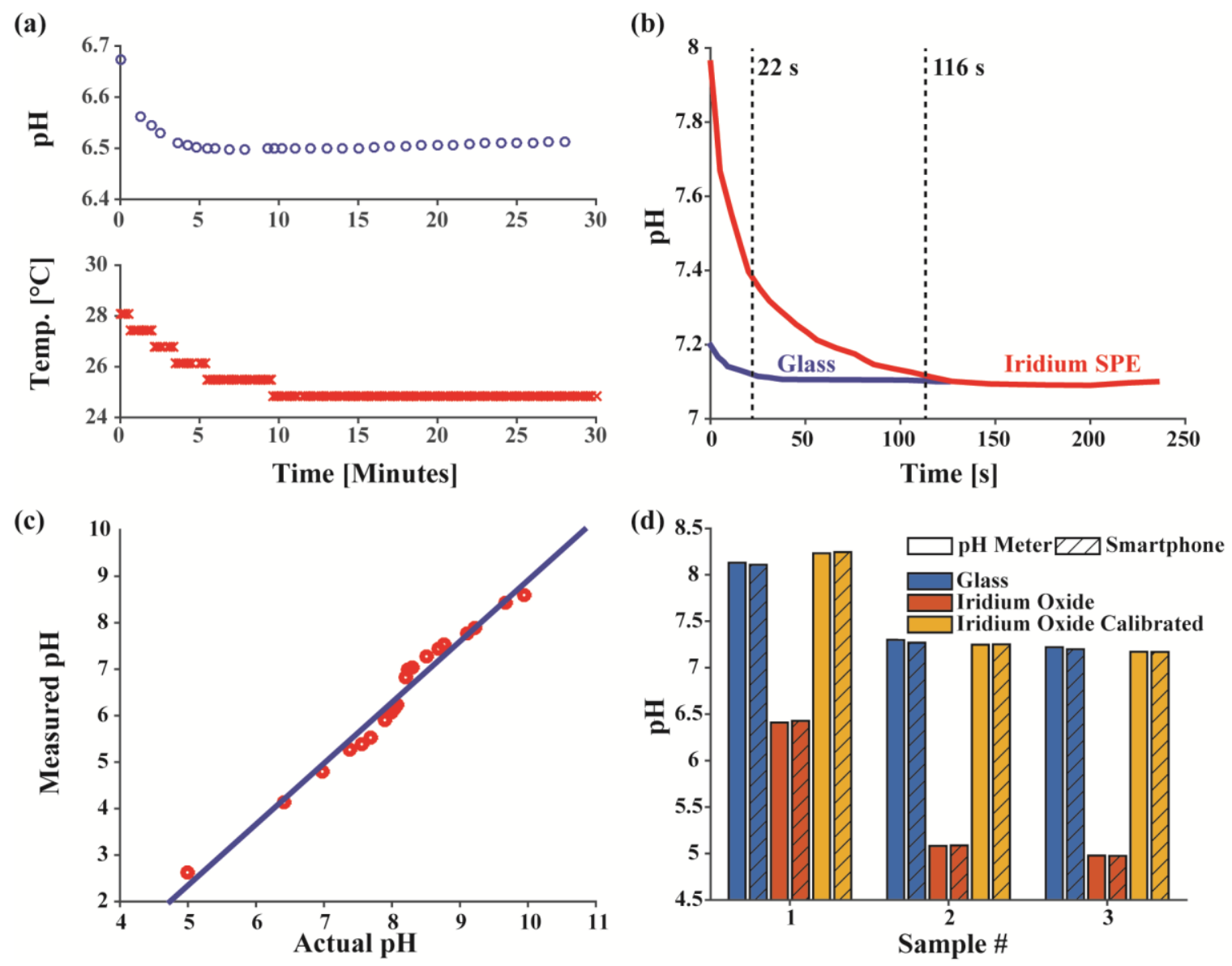

Figure 7. (a) $\mathrm{pH}$ and temperature settling over time of a fresh sputum sample; (b) Settling time of glass and Iridium Oxide electrode; (c) Sputum spiked to different $\mathrm{pH}$ levels measured with both glass and Iridium Oxide electrodes; (d) Actual sputum samples measured and calibrated.

Most importantly, these patient measurements also demonstrate that the entire smartphone-based $\mathrm{pH}$ sensing platform paired with either a standard glass probe or IrOx electrode performs as well as a commercial benchtop meter with a maximum variation between both sets of equipment of $0.031 \mathrm{pH}$. Hence, the smartphone-based $\mathrm{pH}$ sensor can achieve similar accuracy of a laboratory oriented tool, while at the same time being much more portable and amendable to point-of-care applications.

\section{Conclusions}

A POC-oriented, smartphone-based $\mathrm{pH}$ sensor was designed, built, and tested with both standard glass probes and IrOx electrodes for the application of monitoring $\mathrm{pH}$ levels of sputum in patients diagnosed with CF. The electronic peripheral module that attaches to the headphone port of the smartphone measures $\mathrm{pH}$ accurately when compared to a benchtop $\mathrm{pH}$ meter, all while being powered and controlled by the smartphone itself. Furthermore, the point-of-care oriented $\mathrm{pH}$ electrodes were shown to have a high sensitivity comparable to that of glass probes with acceptable amount stability given appropriate calibration. This complete, smartphone-based platform capable of dynamically interfacing with both types of sensors was shown to accurately measure actual sputum samples, which were verified with the benchtop meter. Hence, in terms of portability, accuracy, and flexibility, 
this platform has shown promise for practical at-home $\mathrm{pH}$ monitoring of lung health for $\mathrm{CF}$ patients. In the future, with this flexible platform as a launching pad, more CF biomarker sensors can be added to further improve the specificity and accuracy of this POC tool.

Supplementary Materials: The code for the smartphone application can be found online at https://github.com/ acs009/smartphone-ph. The following are available online at http://www.mdpi.com/1424-8220/17/6/1245/s1, Figure S1: CAD images of 3D printed enclosure for electronic device, Figure S2: Sensor response to temperature variation and calibration of $\mathrm{pH}$, Table S1: Bill of materials, Video S1: Smartphone pH Sensor Demo.

Acknowledgments: This work was partially supported by the National Institutes of Health, Grant UL1TR000100. The content is solely the responsibility of the authors and does not necessarily represent the official views of the NIH. The authors would like to thank the three anonymous patients from whom samples were collected with IRB approval.

Author Contributions: Chengyang Yao, Tom Phelps, A. G. Venkatesh, and Drew A. Hall designed and built the electronics, sensors, and software; All authors conceived and designed the experiments; Alexander Sun, Tom Phelps, A. G. Venkatesh, and Douglas Conrad performed the experiments; Alexander Sun, Tom Phelps, and A. G. Venkatesh analyzed the data; Douglas Conrad, and Drew A. Hall contributed reagents/materials/analysis tools; Alexander Sun wrote the paper.

Conflicts of Interest: The authors declare no conflict of interest. The founding sponsors had no role in the design of the study; in the collection, analyses, or interpretation of data; in the writing of the manuscript, and in the decision to publish the results.

\section{References}

1. Cutting, G.R. Cystic fibrosis genetics: From molecular understanding to clinical application. Nat. Rev. Genet. 2015, 16, 45-56. [CrossRef] [PubMed]

2. Tuchman, L.K.; Schwartz, L.A.; Sawicki, G.S.; Britto, M.T. Cystic Fibrosis and Transition to Adult Medical Care. Pediatrics 2010, 125, 566-573. [CrossRef] [PubMed]

3. Stoltz, D.A.; Meyerholz, D.K.; Welsh, M.J. Origins of Cystic Fibrosis Lung Disease. N. Engl. J. Med. 2015, 372, 351-362. [CrossRef] [PubMed]

4. Metters, J.P.; Kampouris, D.K.; Banks, C.E. Electrochemistry provides a point-of-care approach for the marker indicative of Pseudomonas aeruginosa infection of cystic fibrosis patients. Analyst 2014, 139, 3999-4004. [CrossRef] [PubMed]

5. Eickmeier, O.; Huebner, M.; Herrmann, E.; Zissler, U.; Rosewich, M.; Baer, P.C.; Buhl, R.; Schmitt-Grohé, S.; Zielen, S.; Schubert, R. Sputum biomarker profiles in cystic fibrosis (CF) and chronic obstructive pulmonary disease (COPD) and association between pulmonary function. Cytokine 2010, 50, 152-157. [CrossRef] [PubMed]

6. Weldon, S.; McNally, P.; McElvaney, N.G.; Elborn, J.S.; McAuley, D.F.; Wartelle, J.; Belaaouaj, A.; Levine, R.L.; Taggart, C.C. Decreased Levels of Secretory Leucoprotease Inhibitor in the Pseudomonas-Infected Cystic Fibrosis Lung Are Due to Neutrophil Elastase Degradation. J. Immunol. 2009, 183, 8148-8156. [CrossRef] [PubMed]

7. Mayer-Hamblett, N.; Aitken, M.L.; Accurso, F.J.; Kronmal, R.A.; Konstan, M.W.; Burns, J.L.; Sagel, S.D.; Ramsey, B.W. Association between Pulmonary Function and Sputum Biomarkers in Cystic Fibrosis. Am. J. Respir. Crit. Care Med. 2007, 175, 822-828. [CrossRef] [PubMed]

8. Ojoo, J.C.; Mulrennan, S.A.; Kastelik, J.A.; Morice, A.H.; Redington, A.E. Exhaled breath condensate pH and exhaled nitric oxide in allergic asthma and in cystic fibrosis. Thorax 2005, 60, 22-26. [CrossRef] [PubMed]

9. Carpagnano, G.E.; Barnes, P.J.; Francis, J.; Wilson, N.; Bush, A.; Kharitonov, S.A. Breath condensate $\mathrm{pH}$ in children with cystic fibrosis and asthma*: A new noninvasive marker of airway inflammation? Chest 2004, 125, 2005-2010. [CrossRef] [PubMed]

10. Quittner, A.L.; Sawicki, G.S.; McMullen, A.; Rasouliyan, L.; Pasta, D.J.; Yegin, A.; Konstan, M.W. Erratum to: Psychometric evaluation of the Cystic Fibrosis Questionnaire-Revised in a national, US sample. Qual. Life Res. 2012, 21, 1279-1290. [CrossRef] [PubMed]

11. Quittner, A.L.; Modi, A.C.; Wainwright, C.; Otto, K.; Kirihara, J.; Montgomery, A.B. DEtermination of the minimal clinically important difference scores for the cystic fibrosis questionnaire-revised respiratory symptom scale in two populations of patients with cystic fibrosis and chronic pseudomonas aeruginosa airway infection. Chest 2009, 135, 1610-1618. [CrossRef] [PubMed] 
12. Kupferberg, D.H.; Kaplan, R.M.; Slymen, D.J.; Ries, A.L. Minimal clinically important difference for the UCSD Shortness of Breath Questionnaire. J. Cardpulm. Rehabil. 2005, 25, 370-377. [CrossRef]

13. Eakin, E.G.; Resnikoff, P.M.; Prewitt, L.M.; Ries, A.L.; Kaplan, R.M. Validation of a new dyspnea measure: The ucsd shortness of breath questionnaire. Chest 1998, 113, 619-624. [CrossRef] [PubMed]

14. De Matteis, V.; Cannavale, A.; Blasi, L.; Quarta, A.; Gigli, G. Chromogenic device for cystic fibrosis precocious diagnosis: A "point of care" tool for sweat test. Sens. Actuators B Chem. 2016, 225, 474-480. [CrossRef]

15. Gonzalo-Ruiz, J.; Mas, R.; de Haro, C.; Cabruja, E.; Camero, R.; Alonso-Lomillo, M.A.; Muñoz, F.J. Early determination of cystic fibrosis by electrochemical chloride quantification in sweat. Biosens. Bioelectron. 2009, 24, 1788-1791. [CrossRef] [PubMed]

16. Bonanni, A.; Esplandiu, M.J.; del Valle, M. Impedimetric genosensing of DNA polymorphism correlated to cystic fibrosis: A comparison among different protocols and electrode surfaces. Biosens. Bioelectron. 2010, 26, 1245-1251. [CrossRef] [PubMed]

17. Lagae, L.; Wirix-Speetjens, R.; Liu, C.X.; Laureyn, W.; Borghs, G.; Harvey, S.; Galvin, P.; Ferreira, H.A.; Graham, D.L.; Freitas, P.P.; et al. Magnetic biosensors for genetic screening of cystic fibrosis. IEE Proc. Circuits Devices Syst. 2005, 152, 393-400. [CrossRef]

18. Benuzzi, M.L. S.; Pereira, S.V.; Raba, J.; Messina, G.A. Screening for cystic fibrosis via a magnetic and microfluidic immunoassay format with electrochemical detection using a copper nanoparticle-modified gold electrode. Microchim. Acta 2016, 183, 397-405. [CrossRef]

19. Piraino, F.; Volpetti, F.; Watson, C.; Maerkl, S.J. A Digital-Analog Microfluidic Platform for Patient-Centric Multiplexed Biomarker Diagnostics of Ultralow Volume Samples. ACS Nano 2016, 10, 1699-1710. [CrossRef] [PubMed]

20. Curto, V.F.; Coyle, S.; Byrne, R.; Angelov, N.; Diamond, D.; Benito-Lopez, F. Concept and development of an autonomous wearable micro-fluidic platform for real time $\mathrm{pH}$ sweat analysis. Sens. Actuators B Chem. 2012, 175, 263-270. [CrossRef]

21. Hong, J.I.; Chang, B.-Y. Development of the smartphone-based colorimetry for multi-analyte sensing arrays. Lab Chip 2014, 14, 1725-1732. [CrossRef] [PubMed]

22. Shen, L.; Hagen, J.A.; Papautsky, I. Point-of-care colorimetric detection with a smartphone. Lab Chip 2012, 12, 4240-4243. [CrossRef] [PubMed]

23. Curto, V.F.; Fay, C.; Coyle, S.; Byrne, R.; O’Toole, C.; Barry, C.; Hughes, S.; Moyna, N.; Diamond, D.; Benito-Lopez, F. Real-time sweat $\mathrm{pH}$ monitoring based on a wearable chemical barcode micro-fluidic platform incorporating ionic liquids. Sens. Actuators B Chem. 2012, 171-172, 1327-1334. [CrossRef]

24. Xu, W.; Lu, S.; Chen, Y.; Zhao, T.; Jiang, Y.; Wang, Y.; Chen, X. Simultaneous color sensing of $\mathrm{O}_{2}$ and $\mathrm{pH}$ using a smartphone. Sens. Actuators B Chem. 2015, 220, 326-330. [CrossRef]

25. Oncescu, V.; O'Dell, D.; Erickson, D. Smartphone based health accessory for colorimetric detection of biomarkers in sweat and saliva. Lab Chip 2013, 13, 3232-3238. [CrossRef] [PubMed]

26. Bandodkar, A.J.; Hung, V.W.S.; Jia, W.; Valdés-Ramírez, G.; Windmiller, J.R.; Martinez, A.G.; Ramírez, J.; Chan, G.; Kerman, K.; Wang, J. Tattoo-based potentiometric ion-selective sensors for epidermal $\mathrm{pH}$ monitoring. Analyst 2012, 138, 123-128. [CrossRef] [PubMed]

27. Nemiroski, A.; Christodouleas, D.C.; Hennek, J.W.; Kumar, A.A.; Maxwell, E.J.; Fernández-Abedul, M.T.; Whitesides, G.M. Universal mobile electrochemical detector designed for use in resource-limited applications. Proc. Natl. Acad. Sci. USA 2014, 111, 11984-11989. [CrossRef] [PubMed]

28. Bratov, A.; Abramova, N.; Ipatov, A. Recent trends in potentiometric sensor arrays-A review. Anal. Chim. Acta 2010, 678, 149-159. [CrossRef] [PubMed]

29. Korostynska, O.; Arshak, K.; Gill, E.; Arshak, A. Review Paper: Materials and Techniques for In Vivo pH Monitoring. IEEE Sens. J. 2008, 8, 20-28. [CrossRef]

30. Korostynska, O.; Arshak, K.; Gill, E.; Arshak, A. Review on State-of-the-art in Polymer Based pH Sensors. Sensors 2007, 7, 3027-3042. [CrossRef]

31. Yuqing, M.; Jianrong, C.; Keming, F. New technology for the detection of pH. J. Biochem. Biophys. Methods 2005, 63, 1-9. [CrossRef] [PubMed]

32. Wang, M.; Yao, S.; Madou, M. A long-term stable iridium oxide pH electrode. Sens. Actuators B Chem. 2002, 81, 313-315. [CrossRef] 
33. Marzouk, S.A.; Ufer, S.; Buck, R.P.; Johnson, T.A.; Dunlap, L.A.; Cascio, W.E. Electrodeposited iridium oxide $\mathrm{pH}$ electrode for measurement of extracellular myocardial acidosis during acute ischemia. Anal. Chem. 1998, 70, 5054-5061. [CrossRef] [PubMed]

34. Burke, L.D.; Mulcahy, J.K.; Whelan, D.P. Preparation of an oxidized iridium electrode and the variation of its potential with pH. J. Electroanal. Chem. Interfacial Electrochem. 1984, 163, 117-128. [CrossRef]

35. Lakard, B.; Herlem, G.; Lakard, S.; Guyetant, R.; Fahys, B. Potentiometric pH sensors based on electrodeposited polymers. Polymer 2005, 46, 12233-12239. [CrossRef]

36. Kuo, Y.-S.; Schmid, T.; Dutta, P. Hijacking power and bandwidth from the mobile phone's audio interface. In Proceedings of the First ACM Annual Symposium on Computing for Development, London, UK, 17-18 December 2010.

37. Laksanasopin, T.; Guo, T.W.; Nayak, S.; Sridhara, A.A.; Xie, S.; Olowookere, O.O.; Cadinu, P.; Meng, F.; Chee, N.H.; Kim, J.; et al. A smartphone dongle for diagnosis of infectious diseases at the point of care. Sci. Transl. Med. 2015, 7, 273re1. [CrossRef] [PubMed]

38. Wang, X.; Gartia, M.R.; Jiang, J.; Chang, T.-W.; Qian, J.; Liu, Y.; Liu, X.; Liu, G.L. Audio jack based miniaturized mobile phone electrochemical sensing platform. Sens. Actuators B Chem. 2015, 209, 677-685. [CrossRef]

39. Zhang, D.; Lu, Y.; Zhang, Q.; Liu, L.; Li, S.; Yao, Y.; Jiang, J.; Liu, G.L.; Liu, Q. Protein detecting with smartphone-controlled electrochemical impedance spectroscopy for point-of-care applications. Sens. Actuators B Chem. 2016, 222, 994-1002. [CrossRef]

40. Yao, C.; Sun, A.; Hall, D.A. Efficient Power Harvesting from the Mobile Phone Audio Jack for mHealth Peripherals. In Proceedings of the 2015 IEEE Global Humanitarian Technology Conference (GHTC), Seattle, WA, USA, 8-11 October 2015.

41. Sun, A.; Wambach, T.; Venkatesh, A.G.; Hall, D.A. A low-cost smartphone-based electrochemical biosensor for point-of-care diagnostics. In Proceedings of the 2014 IEEE Biomedical Circuits and Systems Conference (BioCAS), Lausanne, Switzerland, 22-24 October 2014; pp. 312-315.

42. Sun, A.C.; Yao, C.; Venkatesh, A.G.; Hall, D.A. An efficient power harvesting mobile phone-based electrochemical biosensor for point-of-care health monitoring. Sens. Actuators B Chem. 2016, 235, 126-135. [CrossRef] [PubMed]

43. Yao, S.; Wang, M.; Madou, M. A pH Electrode Based on Melt-Oxidized Iridium Oxide. J. Electrochem. Soc. 2001, 148, H29-H36. [CrossRef]

44. Agnew, W.F.; Yuen, T.G.H.; McCreery, D.B.; Bullara, L.A. Histopathologic evaluation of prolonged intracortical electrical stimulation. Exp. Neurol. 1986, 92, 162-185. [CrossRef]

45. Mailley, S.C.; Hyland, M.; Mailley, P.; McLaughlin, J.M.; McAdams, E.T. Electrochemical and structural characterizations of electrodeposited iridium oxide thin-film electrodes applied to neurostimulating electrical signal. Mater. Sci. Eng. C 2002, 21, 167-175. [CrossRef]

46. Dias, N.S.; Carmo, J.P.; da Silva, A.F.; Mendes, P.M.; Correia, J.H. New dry electrodes based on iridium oxide (IrO) for non-invasive biopotential recordings and stimulation. Sens. Actuators Phys. A 2010, 164, $28-34$. [CrossRef]

47. Göbbels, K.; Kuenzel, T.; van Ooyen, A.; Baumgartner, W.; Schnakenberg, U.; Bräunig, P. Neuronal cell growth on iridium oxide. Biomaterials 2010, 31, 1055-1067. [CrossRef] [PubMed]

(C) 2017 by the authors. Licensee MDPI, Basel, Switzerland. This article is an open access article distributed under the terms and conditions of the Creative Commons Attribution (CC BY) license (http://creativecommons.org/licenses/by/4.0/). 